\title{
Efficacy and Safety of Epirubicin Combined with Temozolomide for Treatment of Advanced Leiomyosarcoma
}

\author{
Huijing Tan (D) \\ Lijie Zuo ${ }^{1,2}$ \\ Shutao Ma iD ${ }^{\prime}$ \\ Dingyuan Wang $\mathbb{D D}^{3}$ \\ Rui $\mathrm{Li}^{1,2}$ \\ Yiqi Yang (D) ${ }^{1,2}$ \\ Weili Liu (iD) ${ }^{1,2}$ \\ Yihebali Chi ${ }^{\prime}$ \\ 'Department of Medical Oncology, \\ National Cancer Center/National Clinical \\ Research Center for Cancer/Cancer \\ Hospital, Chinese Academy of Medical \\ Sciences and Peking Union Medical \\ College, Beijing, I0002I, People's \\ Republic of China; ${ }^{2}$ Beijing Chaoyang \\ Sanhuan Cancer Hospital, Beijing, \\ I0002I, People's Republic of China; \\ ${ }^{3}$ Department of Breast Surgery, National \\ Cancer Center/National Clinical \\ Research Center for Cancer/Cancer \\ Hospital, Chinese Academy of Medical \\ Sciences and Peking Union Medical \\ College, Beijing, I0002I, People's \\ Republic of China
}

Background: Leiomyosarcoma (LMS) accounts for 24\% of all soft tissue sarcomas (STSs) and this STS subtype has high metastatic potential. Previous studies indicated the best median progression-free survival (mPFS) time was 9.2 months and the best overall response rate (ORR) was $30.9 \%$. We evaluated the efficacy and safety of epirubicin combined with temozolomide (EPI-TMZ) for treatment of advanced LMS.

Methods: This was a retrospective review of the records of patients with advanced LMS at the National Cancer Center/National Clinical Research Center for Cancer/Cancer Hospital, Chinese Academy of Medical Sciences and Peking Union Medical College. All patients initiated EPI-TMZ treatment between January 2018 and December 2020.

Results: We examined 15 patients who received EPI-TMZ for LMS. This was a first-line treatment in 6 patients, a second- or third-line treatment in 7 patients, and a fourth-line treatment in 2 patients. At the time of data cutoff (April 25, 2021), the median PFS was 10 months, 1 patient had clinical complete response (cCR), 7 had partial response (PR), and 7 had stable disease (SD). The overall response rate (ORR) was $53.3 \%(8 / 15)$ and the disease control rate (DCR) was $100.0 \%(15 / 15)$. The most common treatment-related adverse effects were leukopenia, neutropenia, thrombocytopenia, anemia, nausea, vomiting, fatigue, and oral mucositis. One patient had severe adverse effect (febrile neutropenia), but there were no treatment-related deaths.

Conclusion: EPI-TMZ is potentially effective for treatment of advanced LMS, and the adverse effects appear tolerable. EPI-TMZ provided better outcomes than reported in previous studies of other treatments for advanced LMS.

Keywords: epirubicin, temozolomide, advanced leiomyosarcoma, chemotherapy

\section{Introduction}

Soft tissue sarcomas (STSs) are malignant tumors originating from mesenchymal tissue and account for about $0.8 \%$ of all human malignancies. ${ }^{1}$ There are more than 50 subtypes of STSs and they have a high degree of heterogeneity. When a patient has a single resectable metastasis, radiotherapy with or without chemotherapy is performed before or after surgery. For patients with advanced, unresectable, or systemic metastatic STSs, palliative chemotherapy remains the main treatment regimen. ${ }^{2}$

Leiomyosarcoma (LMS) is a common invasive subtype of STS that has high metastatic potential. LMS accounts for $24 \%$ of all STSs, ${ }^{3}$ is most common in middle-aged and elderly patients, and especially in those who are 40 to 60 yearsold with non-primary immunodeficiency disorders, post-transplantation, and HIV
Correspondence: Yihebali Chi Department of Medical Oncology, National Cancer Center/National Clinical Research Center for Cancer/Cancer Hospital, Chinese Academy of Medical Sciences and Peking Union Medical College, 17 South Panjiayuan Lane, Chaoyang District, Beijing, I0002I, People's Republic of China Tel +861391 075626

Email yihebalichi@hotmail.com 
positive. Some retrospective studies found that palliative chemotherapy for LMS is less effective than for other types of STSs. ${ }^{4}$

There are currently no highly reliable factors for predicting the prognosis of patients with LMS. Most of these patients have tumors in the retroperitoneum (23\%) and gynecological organs (33\%), recurrent hematogenous metastasis can occur, and early distant metastasis is the main form of recurrence. Local recurrence and metastasis can occur from several years to more than ten years after the initial operation.

LMS can be divided into different subtypes according to location: angiomyosarcoma, superficial LMS, and deep soft tissue LMS. ${ }^{5}$ Deep soft tissue LMS is moderately sensitive to chemotherapy, and the primary tumor often occurs in the retroperitoneum, pelvis, large blood vessels, and extremities. Primary retroperitoneal LMS (RLMS) is a common deep soft tissue LMS and one of the major types of retroperitoneal STSs. ${ }^{6}$ The onset of RLMS is often asymptomatic, and when identified the tumor is typically adherent to adjacent tissue. RLMS can easily invade large blood vessels, leading to a low rate of complete resection, so these patients have poorer prognosis than those with other types of LMS. RLMS can recur locally and can also metastasize to the liver and lungs, and the overall 5-year survival rate of these patients is about 50 to $60 \% .{ }^{1}$ The Memorial Sloan-Kettering Cancer Center reported that the incidence of new cases of retroperitoneal sarcoma in China was 9000 to 10,000 per year. ${ }^{1,7}$

At present, surgical resection plays the main role in comprehensive treatment of LMS. Anthracycline-based therapy, with or without ifosfamide, are still first-line standard chemotherapy regimens for advanced LMS, but the efficacy is poor. In addition, uterine LMS (uLMS), a rare primary malignant tumor of the uterus $(<5 \%)$, is more sensitive to chemotherapy. Although only a small proportion of LMS patients have high-grade uLMS, about $50 \%$ of those with high-grade uLMS experience relapse within 2 years. This relapse can occur in the pelvic region or more commonly in extrapelvic regions (abdomen, hepatopulmonary metastasis, bone metastasis, distant subcutaneous or soft tissue metastasis). BRCAness, a DNA homologous recombination repair defect, is more common in uLMS, so this LMS subtype obviously has greater defects in DNA repair. In addition, uLMS is also distinguished from other subtypes by DNA methylation and other genetic characteristics. Factors that affect the prognosis of advanced uLMS include age greater than 62 years-old, tumor size greater than $4 \mathrm{~cm}$, tumor necrosis, high Fédération Nationale des Centres de Lutte Contre le Cancer (FNCLCC) grade, primary site, vascular invasion, and previous resection. ${ }^{8,9}$

Temozolomide (TMZ) is a new oral alkylating agent that is mainly used for treatment of glioblastoma. When TMZ enters the systemic circulation at a normal physiological $\mathrm{pH}$, it is rapidly hydrolyzed into the active product 3-methyl-(triazine-1-) imidazole-4-formamide (MTIC). MTIC alkylates the sixth oxygen atom and the seventh nitrogen of guanine residues in DNA, leading to cell death. Compared with dacarbazine, cytochrome P450 plays only a minor role in the metabolism of TMZ, and liver damage, including fatal liver failure, is rare. However, TMZ and dacarbazine have comparable efficacies, ${ }^{10}$ and there is evidence that TMZ improves the overall survival rate and progression-free survival (PFS) of patients with advanced metastatic melanoma. ${ }^{11,12} \mathrm{TMZ}$ has acceptable safety, provides quality of life benefits, can penetrate the blood-brain barrier, and is easy to administer. Thus, TMZ may have potential for the treatment of LMS.

The results of several Phase II clinical studies ${ }^{13-15}$ indicated that TMZ monotherapy was an effective treatment for advanced STS after classic first-line chemotherapy, especially in patients with advanced non-surgical resection of uterine or non-uterine LMS. In particular, compared with the whole STS population, patients who received TMZ had better outcomes in terms of objective response rate (ORR, $18 \%$ vs $8 \%$ ), stable disease (SD, $27 \%$ vs $8 \%$ ), disease control rate (DCR, $45 \%$ vs $16 \%$ ), median PFS (mPFS, 3.9 vs 2.0 months), and median overall survival (mOS, 30.8 vs 13.0 months). Most of the adverse reactions were grade 1 or 2 , and the grade 3 toxicities included nausea, anemia, fatigue, elevated alkaline phosphatase level, and non-neutropenic fever (1 case each).$^{13}$ Other research reported that the adverse effects of epirubicin (EPI), especially cardiac and blood toxicities, were less than those of doxorubicin. ${ }^{16}$ These observations led us to hypothesize that EPI and TMZ (EPITMZ) may be an effective and tolerable treatment for advanced LMS.

We retrospectively examined the safety and efficacy of a chemotherapy regimen consisting EPI-TMZ for the treatment of patients with advanced LMS as an initial effort to identify a novel, effective, and safe treatment for these patients. 


\section{Materials and Methods}

\section{Patient Enrolment}

This was a single-center, retrospective clinical study conducted at the Cancer Hospital of the Chinese Academy of Medical Sciences in Beijing. The protocol was approved by the Chinese Academy of Medical Sciences Cancer Hospital Institutional Review Board at the participating institution, and complied with good clinical practice guidelines and the Declaration of Helsinki. Due to the retrospective nature of this study, the requirement for informed consent was waived. All data were anonymized to maintain patient confidentiality. Fifteen patients who met the following criteria were selected: histopathologically confirmed and surgically unresectable or metastatic LMS, availability of follow-up imaging data, anthracycline combination chemotherapy, EPI-TMZ treatment that started between January 2018 to December 2020, at least one measurable lesion based on Response Evaluation Criteria in Solid Tumors (RECIST) version 1.1, performance status (PS) score of 2 or less in the Eastern Cooperative Oncology Group (ECOG) scale, and age of at least 18 years-old. The additional recorded clinical data were sex (14 [93.3\%] female), primary site (6 [40.0\%] retroperitoneum, $6[40.0 \%]$ uterus, $2[13.3 \%]$ inferior vena cava, 1 [6.7\%] colonic mesentery), metastasis site (13 [86.7\%] lung, 10 [66.7\%] liver, 3 [20.0\%] bone), and previous treatment (14 [93.3\%] surgery, 1 [6.7\%] radiotherapy, and $9[60.0 \%]$ chemotherapy).

\section{Chemotherapy Regimen}

All 15 patients received EPI-TMZ. Six patients received this treatment as a first-line regimen, 7 as a second-line regimen, and 2 as a fourth-line regimen. Injections of polyethylene glycol granulocyte colony stimulating factor were given selectively if necessary. Oral TMZ (200 or $300 \mathrm{mg}$ ) was administered each day on days 1 to 5 , and intravenous EPI $\left(60 \mathrm{mg} / \mathrm{m}^{2}\right)$ was administered on days 1 to 4, with even distribution over these $96 \mathrm{~h}$. There was 1 therapy cycle every 3 weeks. Drug use continued until the patient experienced progressive disease (PD) or intolerable adverse effects (AEs). The dosage was adjusted according to adverse reactions.

\section{Outcome Indicators}

Efficacy was evaluated every two treatment cycles. According to RECIST version 1.1, the results were as recorded as complete response (CR), partial response
$(\mathrm{PR})$, stable disease (SD), or progressive disease (PD). The overall response rate (ORR) was the sum of CR and $\mathrm{PR}$, and the disease control rate (DCR) was the sum of $\mathrm{CR}, \mathrm{PR}$, and SD. The related adverse reactions were evaluated according to version 4 of the Common Drug Toxicity classification standard of the US National Cancer Institute (NCI-CTC 4.0). The main endpoints were progression-free survival (PFS), overall response rate (ORR), and adverse effects (AEs). Statistical analyses were performed using $\mathrm{R}$ software version 4.0.1 or GraphPad Prism software version 9.0.2.

\section{Results \\ Patient Characteristics}

There were 15 patients ( 1 male and 14 females), the median age was 53 years-old (range: 27-63), and the primary tumor was in the uterus $(n=6)$, retroperitoneum $(n=6)$, inferior vena cava $(n=2)$, or colonic mesentery $(n=1)$.

\section{Treatment Regimens}

Twelve patients $(80.0 \%)$ received all 8 cycles of the intensive chemotherapy. There were two subsequent basic maintenance therapies. Monodrug maintenance therapy consisted of TMZ alone (dosing as in the primary therapy), and two-drug maintenance therapy consisted of TMZ (dosing as in the primary therapy) with oral thalidomide $(100 \mathrm{mg} /$ day from days $1-7,200 \mathrm{mg} /$ day from days 8 $14,300 \mathrm{mg}$ /day from day 15 as long as the condition did not progress), with one cycle every 3 weeks. Among all 15 patients, there was a median of 11 total (intensive and maintenance) cycles (range: 5-24). At the time of data cutoff (April 25, 2021), 3 patients were still receiving maintenance treatment.

\section{Treatment Efficacy}

At the time of analysis, the median PFS was 10 months, with 1 case classified as clinical complete response (cCR, 6.6\%), 7 cases as PR (46.7\%), and the other 7 cases as SD (46.7\%). The ORR was $53.3 \%$ ( 8 cases) and the DCR was $100.0 \%$ ( 15 cases). For the 6 patients who received no previous frontline treatment, the ORR was $66.7 \%$ (4 cases), the DCR was $100.0 \%$ (6 cases), and the mPFS was 9.25 months. For the 7 patients who received EPITMZ as a second or third-line regimen, the ORR was $42.9 \%$ ( 3 cases), the DCR was $100.0 \%$ ( 7 cases), and the mPFS was 10 months. For the two patients who received 
EPI-TMZ as a fourth-line treatment, the ORR was $50.0 \%$ (1 case), the DCR was $100.0 \%$ (2 cases), and mPFS was 9.5 months.

None of the differences we observed in PFS (Table 1) and ORR (Table 2) were significant, and the small sample size precluded further examination. Nonetheless, we observed a tendency for worse outcome in patients with a retroperitoneal origin (median PFS: 8 months; 95\% CI:
4-16) in comparison with those who had origins at other sites (median PFS: 16 months; 95\% CI: 10-22; HR: 0.4; 95\% CI: 0.06-2.58). There was also a tendency for a better ORR in patients with $\mathrm{Ki}-67$ positivity levels above $50 \%$ (100\% vs $30 \%)$.

Overall, the median PFS was 10 months (Figure 1), the average percent maximal sum of the diameters of the target lesions decreased by $33.3 \%$ (Figure 2), the

Table I Univariate Cox Proportional-Hazards Model of the Effect of Different Characteristics on Progression-Free Survival

\begin{tabular}{|c|c|c|c|}
\hline Variable & HR (95\% Cl) & $p$-value & Median PFS, Months $(95 \% \mathrm{Cl})$ \\
\hline \multicolumn{4}{|l|}{ Age, years } \\
\hline$<60$ & I (reference) & & $8(3-21)$ \\
\hline$\geq 60$ & $0.97(0.9-1.05)$ & 0.504 & $10(9-16)$ \\
\hline \multicolumn{4}{|l|}{ Primary site } \\
\hline Retroperitoneum & I (reference) & & $8(4-16)$ \\
\hline Non-retroperitoneal & $0.56(0.12-2.58)$ & 0.457 & $10(3-21)$ \\
\hline Uterus & $0.77(0.14-4.19)$ & 0.761 & $10(3-16)$ \\
\hline Other & $0.40(0.06-2.58)$ & 0.338 & $16(10-22)$ \\
\hline \multicolumn{4}{|l|}{ Tumor size, mm } \\
\hline$\leq 100$ & I (reference) & & $12(6-2 \mid)$ \\
\hline$>100$ & $4.62(0.63-33.8)$ & 0.132 & $4(3-10)$ \\
\hline \multicolumn{4}{|l|}{ Lung metastasis } \\
\hline No & I (reference) & & $10(4-16)$ \\
\hline Yes & $1.16(0.14-9.84)$ & 0.889 & $10(3-21)$ \\
\hline \multicolumn{4}{|l|}{ Hepatic metastasis } \\
\hline No & I (reference) & & $10(6-16)$ \\
\hline Yes & $0.49(0.12-2.02)$ & 0.325 & $10(3-21)$ \\
\hline \multicolumn{4}{|l|}{ Bone metastasis } \\
\hline No & I (reference) & & $9(3-20)$ \\
\hline Yes & $1.07(0.25-4.56)$ & 0.928 & $12(10-17)$ \\
\hline \multicolumn{4}{|l|}{ Number of operations } \\
\hline$\leq 1$ & I (reference) & & $10(5-2 \mid)$ \\
\hline$>1$ & $2.91(0.47-18.14)$ & 0.254 & $7(3-12)$ \\
\hline \multicolumn{4}{|l|}{ Previous drug therapy } \\
\hline No & I (reference) & & $9(3-2 I)$ \\
\hline Yes & $2.22(0.44-11.1)$ & 0.332 & $10(4-17)$ \\
\hline \multicolumn{4}{|l|}{ Frontline TKI treatment } \\
\hline No & I (reference) & & $10(3-21)$ \\
\hline Yes & $1.78(0.39-8.21)$ & 0.457 & $9(6-16)$ \\
\hline \multicolumn{4}{|l|}{ Postoperative radiotherapy } \\
\hline No & I (reference) & & $10(3-20)$ \\
\hline Yes & $1.5(0.16-13.67)$ & 0.719 & $12(12-12)$ \\
\hline \multicolumn{4}{|l|}{ Number of previous frontline treatments } \\
\hline None & I (reference) & & $10(4-17)$ \\
\hline One or more & $0.45(0.09-2.26)$ & 0.332 & $9(3-2 I)$ \\
\hline
\end{tabular}

Abbreviations: $\mathrm{HR}$, hazard ratio; PFS, progression-free survival; $\mathrm{Cl}$, confidence interval; TKI, tyrosine kinase inhibitor. 
Table 2 Effect of Different Baseline Characteristics on Overall Response Rate

\begin{tabular}{|c|c|}
\hline Characteristics & ORR (\%) \\
\hline \multicolumn{2}{|l|}{ Primary site } \\
\hline Retroperitoneum & $50 \%(3 / 6)$ \\
\hline Uterus & $50 \%(3 / 6)$ \\
\hline Other & $66.7 \%(2 / 3)$ \\
\hline \multicolumn{2}{|l|}{ Ki-67 positivity } \\
\hline$>50 \%$ & $100 \%(4 / 4)$ \\
\hline$\leq 50 \%$ & $30 \%(3 / 10)$ \\
\hline \multicolumn{2}{|l|}{ Tumor size, mm } \\
\hline$>100$ & $60 \%(3 / 5)$ \\
\hline$\leq 100$ & $44.4 \%(4 / 9)$ \\
\hline \multicolumn{2}{|l|}{ Lung metastasis } \\
\hline Yes & $46.2 \%(6 / 13)$ \\
\hline No & $100 \%(2 / 2)$ \\
\hline \multicolumn{2}{|l|}{ Hepatic metastasis } \\
\hline Yes & $60 \%(6 / 10)$ \\
\hline No & $40 \%(2 / 5)$ \\
\hline \multicolumn{2}{|l|}{ Bone metastasis } \\
\hline Yes & $33.3 \%(1 / 3)$ \\
\hline No & $58.3 \%(7 / 12)$ \\
\hline \multicolumn{2}{|l|}{ Number of operations } \\
\hline$>1$ & $33.3 \%(2 / 6)$ \\
\hline$\leq 1$ & $66.7 \%(6 / 9)$ \\
\hline \multicolumn{2}{|l|}{ Previous drug therapy } \\
\hline Yes & $44.4 \%(4 / 9)$ \\
\hline No & $66.7 \%(4 / 6)$ \\
\hline \multicolumn{2}{|l|}{ Frontline TKI treatment } \\
\hline Yes & $40 \%(2 / 5)$ \\
\hline No & $60 \%(6 / 10)$ \\
\hline
\end{tabular}

Abbreviations: ORR, overall response rate; TKI, tyrosine kinase inhibitors.

average percent change in the target lesion from baseline decreased by $23.5 \%$ (Figure 3), and the average total treatment time was 10.4 months (Figure 4). A hazard ratio plot indicated that none of the analyzed factors were associated with PFS (Figure 5).

\section{Adverse Effects}

The most common treatment-related adverse effects were leukopenia $(26.7 \%)$, neutropenia $(20.0 \%)$, thrombocytopenia $(13.3 \%)$, anemia $(6.7 \%)$, nausea $(46.7 \%)$, vomiting (33.3\%), fatigue $(20.0 \%)$, and oral mucositis $(6.7 \%)$, and most of these were in the tolerable range (data not shown). One patient had a severe adverse effect (febrile neutropenia), but there were no treatment-related deaths.

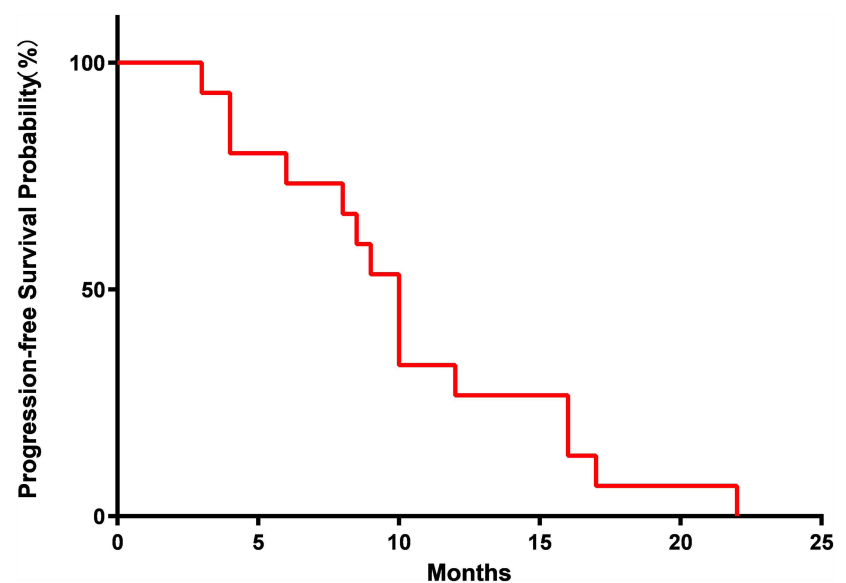

Figure I Probability of progression-free survival (median: 10 months).

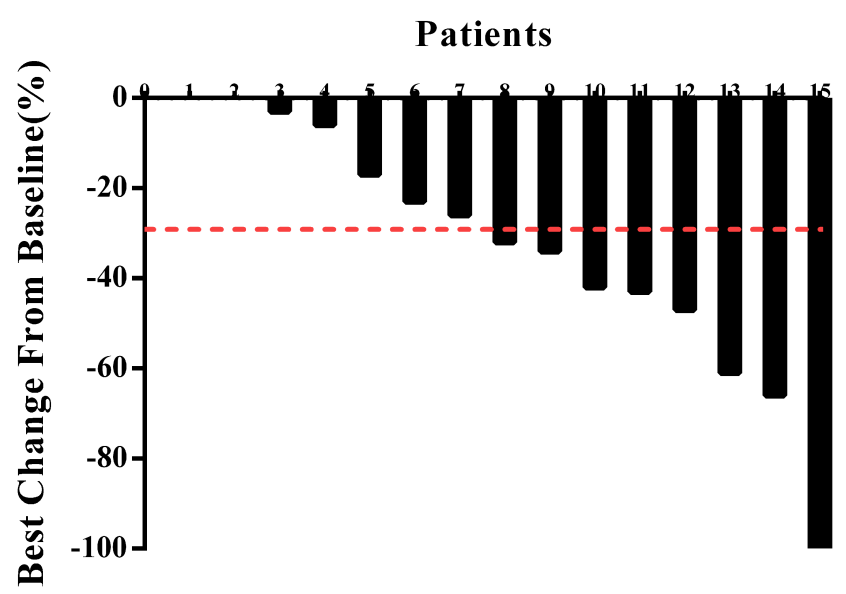

Figure 2 Maximal percent change in the sum of the diameters of target lesions from baseline in each of the 15 patients (mean: 33.3\%).

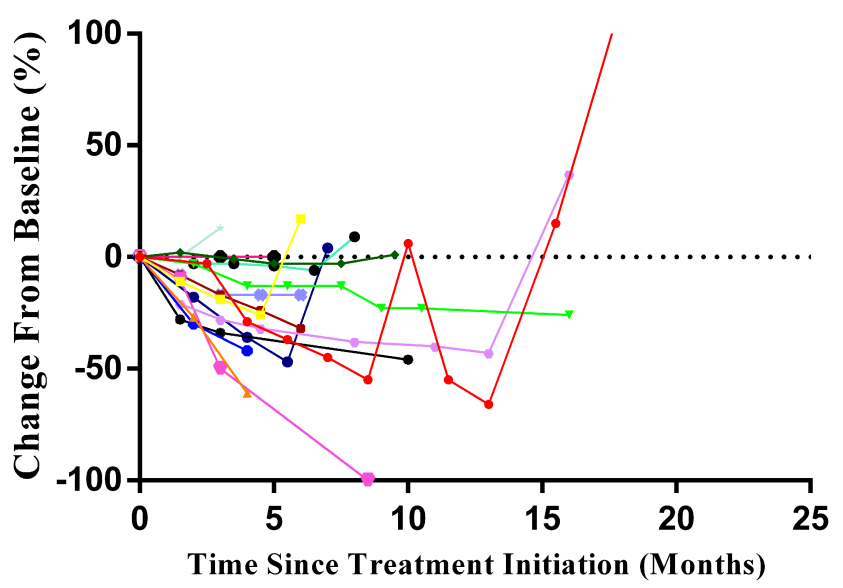

Figure 3 Percent change in the diameter of the target lesion since initiation of treatment in each of the 15 patients (mean: $23.5 \%$ ). 


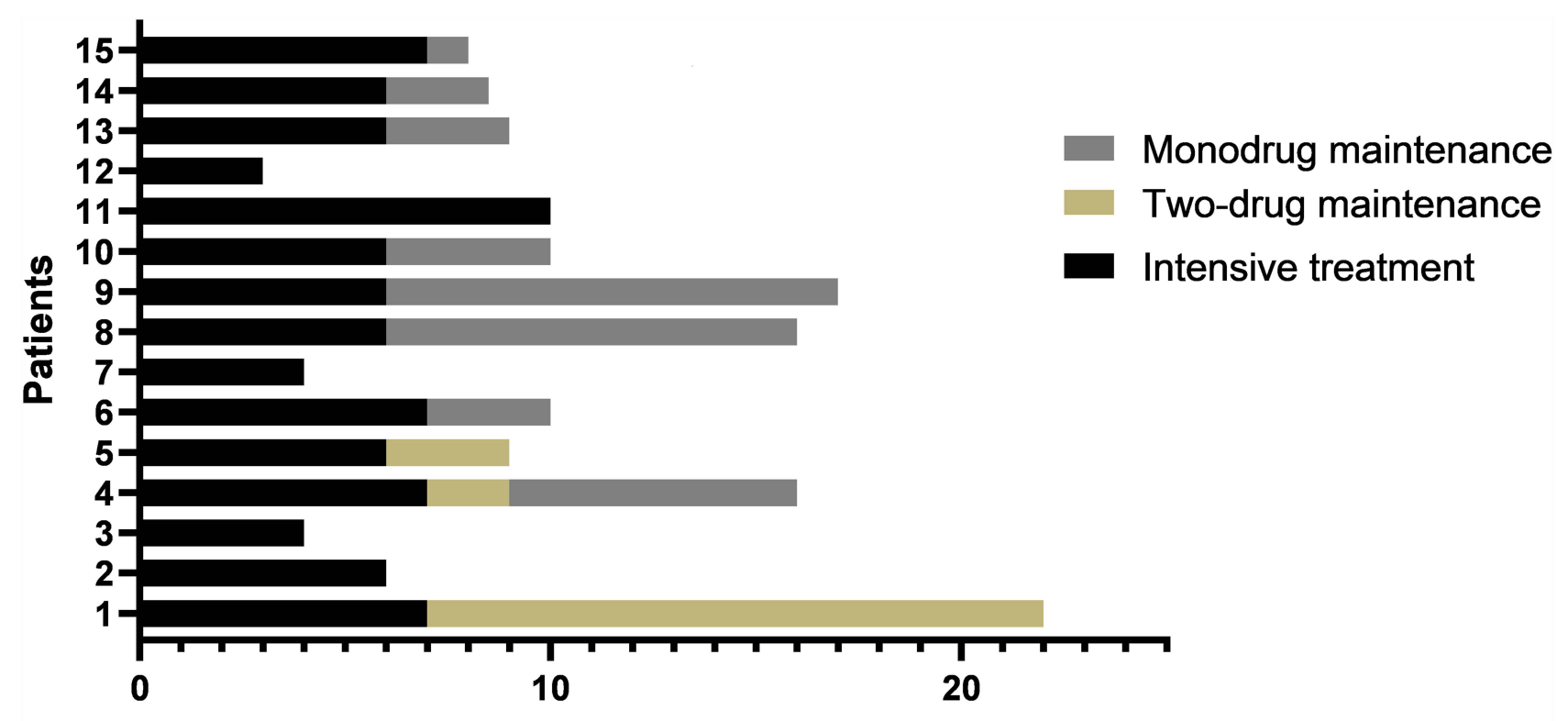

Time Since Treatment Initiation (Months)

Figure 4 Durations of intensive EPI-TMZ treatment (mean: 6.1 months) and maintenance treatment (mean: 4.1 months) in each of the 15 patients. Drug regimens are described in the Methods and Results.

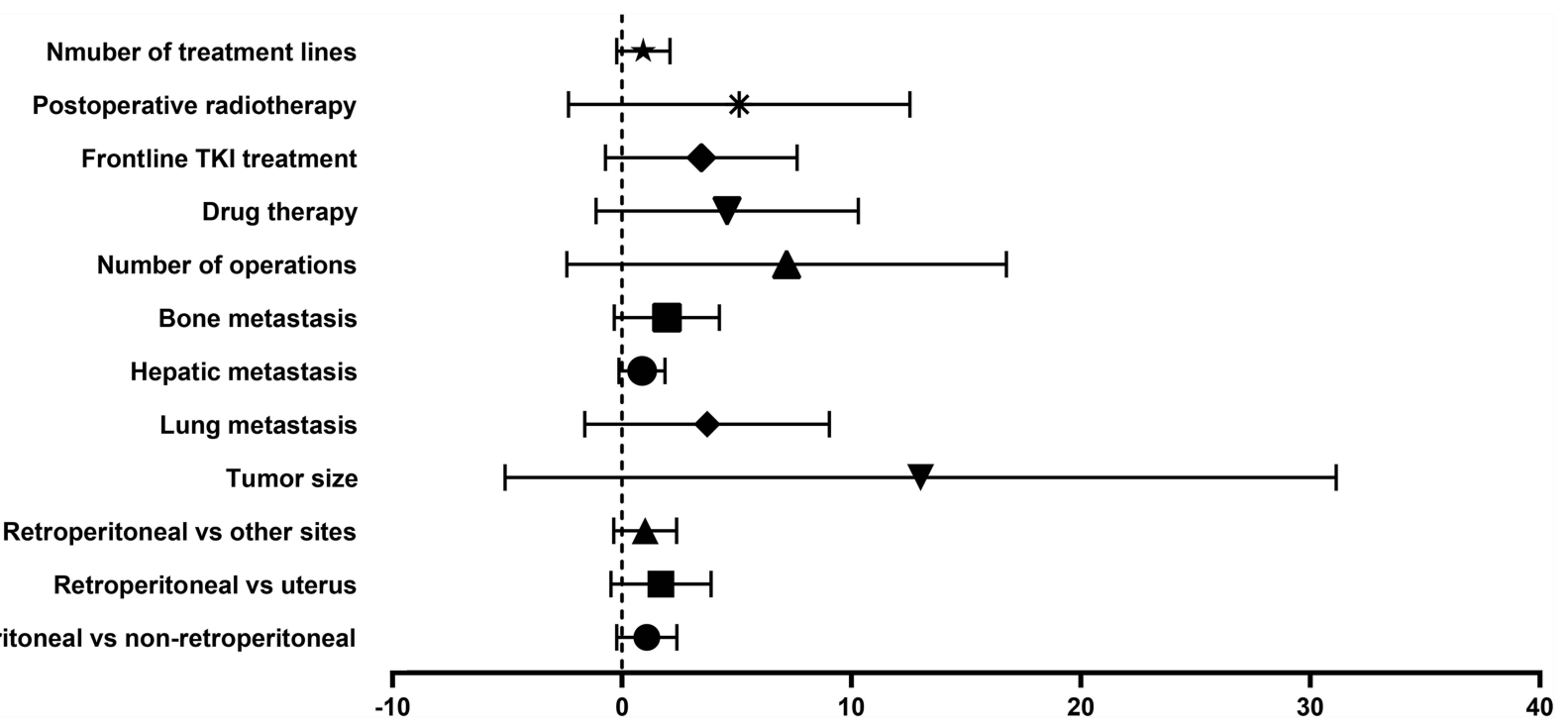

Figure 5 Effect of different factors on the hazard ratio for PFS.

Symptomatic support effectively alleviated all adverse effects.

\section{Discussion}

To our best knowledge and based on historical results (see below), this retrospective study achieved the best PFS and ORR for treatment for advanced LMS. At the time of data cutoff (April 25, 2021), the median PFS was 10 months, the ORR was $53.3 \%$, and the DCR was $100.0 \%$. For patients receiving EPI-TMZ as a first-line therapy, the ORR was $66.7 \%$, the DCR was $100.0 \%$, and the mPFS was 9.25 months; for patients receiving EPI-TMZ as a second- or third-line therapy, the ORR was $42.9 \%$, the DCR was $100.0 \%$, and the mPFS was 10 months. When necessary, we selectively administered polyethylene glycol granulocyte colony-stimulating factor (G-CSF) as supportive treatment, and this likely reduced the incidence of severe myelosuppression and improved the safety of 
EPI-TMZ chemotherapy. Adverse effects in our patients were mainly grade 1 or 2 , indicating that EPI-TMZ chemotherapy was safe for patients with advanced LMS. Our further assessments of the effects of follow-up treatment and overall survival are in progress.

Previous studies examined anthracycline-based regimens, with or without ifosfamide, as standard first-line chemotherapy regimens for metastatic, recurrent, or inoperable LMS. Researchers have not yet identified any highly effective second-line chemotherapy drug or regimen after failure of first-line chemotherapy for advanced LMS. Gemcitabine (GEM), dacarbazine (DTIC), TMZ, trabectedin, and eribulin can be used for second-line single-drug chemotherapy regimens, and GEM with docetaxel (TXT) or GEM with DTIC for combined regimens. Anthracycline-based therapies combined with trabectedin and DTIC can be considered, but the prognosis is still poor. The results of several phase II clinical studies of TMZ monotherapy for treatment of STS ${ }^{13-15}$ indicated this was effective for advanced STS after classic first-line chemotherapy. Moreover, the adverse effects of EPI, especially cardiac and blood toxicities, were less than those of doxorubicin. ${ }^{16}$ This led us to consider a chemotherapy regimen of EPI-TMZ for treatment of advanced LMS.

At the genomic level, LMS is characterized by complex and uncertain genetic variations. TP53 (49\%), RBI (27\%), and $\operatorname{ATRX}(24 \%)$ are the most commonly mutated genes in LMS, and compared with other types of soft tissue sarcomas, LMS has greater change in the homologous recombination pathway. Although the genes driving this condition are unknown, there is abnormal enrichment of some signaling pathways. This suggests the potential for usage of precise targeted antivascular therapies, PARP inhibitors, or mTOR signaling inhibitors. The combined use of immune checkpoint inhibitors and other drugs should be considered. Systemic chemotherapy and molecular targeted therapies are the main methods used to treat advanced LMS, especially in patients with systemic metastatic LMS. Targeted therapy is usually used as a secondline treatment for unresectable or advanced STSs. When the classic chemotherapy treatment fails and when there are contraindications to chemotherapy or when the patient refuses chemotherapy, pazopanib, anlotinib, or regorafenib can be used as second-line treatments. Sunitinib and sorafenib may also be recommended if patients want to participate in clinical trials.

Our major results (ORR of $53.3 \%$ and mPFS of 10 months) indicated that an EPI-TMZ regimen provided better results than previously reported for first-line classic chemotherapy or second-line targeted therapy. The largest retrospective study of the treatment of advanced LMS using a first-line chemotherapy regimen was from European Organization for Research and Treatment of Cancer Soft Tissue and Bone Sarcoma Group, which challenged the classic first-line chemotherapy regimens. ${ }^{17}$ Their results showed that doxorubicin combined with dacarbazine (DoDa) was effective in the treatment of advanced LMS, in that it led to better PFS and ORR than doxorubicin combined with ifosfamide (DI) or doxorubicin alone (Do). In particular, the DoDa group had a mPFS of 9.2 months, an ORR of $30.9 \%$, and an mOS of 36.8 months. As a second-line targeted therapy, the results a Phase III study of pazopanib for metastatic STS showed that this agent significantly increased the median PFS (4.6 months vs 1.6 months) compared with placebo. ${ }^{18} \mathrm{~A}$ phase II study of anlotinib as a second-line treatment for advanced STS reported that patients with LMS had a response rate of $7.7 \%$ and an mPFS of 11.0 months; ${ }^{19}$ another placebo-controlled phase IIB study of anlotinib reported the mPFS of patients with LMS was 5.83 months. ${ }^{20}$ A randomized phase II trial of regorafenib as a treatment for LMS found that the median PFS was 3.7 months, the ORR was $0 \%$, and the DCR was $85.7 \%{ }^{21}$

Therefore, our study of using EPI-TMZ as a treatment for advanced LMS suggests this treatment has promising efficacy and that the adverse effects are tolerable. However, this was a very small single-center study, the study subjects were predominantly female, and we did not perform direct comparisons with a group receiving alternative treatment(s). In addition, we may have overestimated the PFS because the COVID-19 pandemic led to longer time intervals between computed tomography scans, which may have delayed our detection of disease progression.

The tendency for a better outcome in patients with uLMS should be highlighted. Anthracycline-based regimens are front line treatments for LMS, but there is insufficient laboratory evidence or basic research supporting the use of TMZ. One study examined the expression of $\mathrm{O}^{6}$-methylguanine DNA methyltransferase (MGMT) in 6 patients and reported the PFS of $M G M T$-negative patients was 18.5 months, and that of $M G M T$-positive patients was only 3 months. ${ }^{22}$ Thus, expression of MGMT in uLMS negatively correlated with the efficacy of TMZ and patient survival time. Other research reported that assessment of $M G M T$ expression may help to identify a subset of patients 
that respond best to this therapy. ${ }^{23}$ However, these previous results need to be confirmed by large-scale clinical studies.

Recent research identified uLMS as a sarcoma subtype characterized by BRCAness (defective homologous recombination repair pathway due to loss of BRCA1 function), and demonstrated marked and strengthened activity for the PARP inhibitor olaparib in combination with the alkylating agent TMZ in uLMS models. ${ }^{24}$ In subsequent sample-expanded prospective studies, we will further examine the potential mechanism of this effect by assessment of changes in genomic stability, DNA methylation, MGMT status, the mismatch repair (MMR) system, BRCAness, activity of $\mathrm{p} 53,{ }^{25}$ genomic sequences, immunohistochemical expression, and total mutation burden.

\section{Conclusion}

Our study demonstrated that chemotherapy consisting of EPI-TMZ has potential as a new strategy for the treatment of advanced LMS. It is necessary to perform prospective, large-scale, multicenter clinical studies with appropriate controls to determine the practical value EPI-TMZ as a treatment for advanced LMS, and to optimize its use as a first-line chemotherapy regimen for these patients. The goal of this treatment should be significant tumor shrinkage, prolonging the PFS and OS, and improving the life quality of those patients.

Evaluation of the methylation status of the $M G M T$ promoter may help to determine whether a patient can benefit from $\mathrm{TMZ}$ treatment. To identify the potential therapeutic benefits in patients who respond to $\mathrm{TMZ}$, investigations of the mechanisms of this response and resistance to this drug are needed. Continuing efforts are needed to identify alterations in cell function and corresponding downstream pathways after treatment, such as changes in cell proliferation, apoptosis, and tumor size. Ongoing research that is analyzing the characteristics of sarcomas may provide new options for the specific treatment of this rare and aggressive neoplasm.

\section{Ethics Approval and Informed Consent}

This was a single-center, retrospective clinical study conducted at the Cancer Hospital of the Chinese Academy of Medical Sciences in Beijing. The protocol was approved by the Chinese Academy of Medical Sciences Cancer Hospital Institutional Review Board at the participating institution, and complied with good clinical practice guidelines and the Declaration of Helsinki.

\section{Consent for Publication}

Due to the retrospective nature of this study, the requirement for informed consent was waived. All data were anonymized to maintain confidentiality.

\section{Author Contributions}

Yihebali Chi: Conceptualization, Formal analysis, Methodology, Project administration, Resources, Supervision, Validation, Writing - review \& editing; Huijing Tan: Data curation, Formal analysis, Investigation, Project administration, Software, Validation, Visualization, Writing - original draft, Writing - review \& editing; Lijie Zuo: Data curation, Investigation, Project administration, Resources; Shutao Ma: Data curation, Formal analysis, Writing - review \& editing; Dingyuan Wang: Data curation, Software, Visualization; Rui Li: Data curation, Resources, Writing - review \& editing; Yiqi Yang: Data curation, Resources, Writing review \& editing; Weili Liu: Data curation, Resources, Writing - review \& editing. All authors contributed to data analysis, drafting or revising the article, have agreed on the journal to which the article will be submitted, gave final approval of the version to be published, and agree to be accountable for all aspects of the work.

\section{Funding}

There is no funding to report.

\section{Disclosure}

The authors declare that they have no conflicts of interest.

\section{References}

1. Siegel RL, Miller KD, Jemal A. Cancer statistics, 2018. CA Cancer J Clin. 2018;68(1):7-30. doi:10.3322/caac.21442

2. Liu W, Jiang Q, Zhou Y. Advances of systemic treatment for adult soft-tissue sarcoma. Chin Clin Oncol. 2018;7(4):42. doi:10.21037/ cco.2018.08.02

3. Toro JR, Travis LB, Wu HJ, Zhu K, Fletcher CD, Devesa SS. Incidence patterns of soft tissue sarcomas, regardless of primary site, in the surveillance, epidemiology and end results program, 1978-2001: an analysis of 26,758 cases. Int $J$ Cancer. 2006;119(12):2922-2930. doi:10.1002/ijc.22239

4. Van Glabbeke M, Verweij J, Judson I, Nielsen OS. Progression-free rate as the principal end-point for phase II trials in soft-tissue sarcomas. Eur J Cancer. 2002;38(4):543-549. doi:10.1016/S09598049(01)00398-7

5. Wile AG, Evans HL, Romsdahl MM. Leiomyosarcoma of soft tissue: a clinicopathologic study. Cancer. 1981;48(4):1022-1032. doi:10.1002/ 1097-0142(19810815)48:4<1022::AID-CNCR2820480429>3.0.CO;2-5 
6. Nathan H, Raut CP, Thornton K, et al. Predictors of survival after resection of retroperitoneal sarcoma: a population-based analysis and critical appraisal of the AJCC staging system. Ann Surg. 2009;250 (6):970-976. doi:10.1097/SLA.0b013e3181b25183

7. Brennan MF, Antonescu CR, Maki RG. Management of Soft Tissue Sarcoma. 2nd ed. New York: Springer; 2016:3-17.

8. Gustafson P, Willén H, Baldetorp B, Fernö M, Akerman M, Rydholm A. Soft tissue leiomyosarcoma. A population-based epidemiologic and prognostic study of 48 patients, including cellular DNA content. Cancer. 1992;70(1):114-119. doi:10.1002/1097-0142(19920701)70:1<114::AID-CNCR2820700119>3.0.CO;2-U

9. Farshid G, Goldblum J, Weiss SW. Leiomyosarcoma of soft tissue: a tumor of vascular origin with multivariate analysis of outcome. Mod Pathol. 2003;16(8):778-785. doi:10.1097/01.MP.0000083648.45923.2B

10. Patel PM, Suciu S, Mortier L, et al. Extended schedule, escalated dose temozolomide versus dacarbazine in stage IV melanoma: final results of a randomised phase III study (EORTC 18032). Eur J Cancer. 2011;47 (10):1476-1483. doi:10.1016/j.ejca.2011.04.030

11. Middleton MR, Grob JJ, Aaronson N, et al. Randomized phase III study of temozolomide versus dacarbazine in the treatment of patients with advanced metastatic malignant melanoma. J Clin Oncol. 2000;18(1):158-166. doi:10.1200/JCO.2000.18.1.158

12. Kiebert GM, Jonas DL, Middleton MR. Health-related quality of life in patients with advanced metastatic melanoma: results of a randomized phase III study comparing temozolomide with dacarbazine. Cancer Invest. 2003;21(6):821-829. doi:10.1081/CNV-120025084

13. Talbot SM, Keohan ML, Hesdorffer M, et al. A phase II trial of temozolomide in patients with unresectable or metastatic soft tissue sarcoma. Cancer. 2003;98(9):1942-1946. doi:10.1002/cncr.11730

14. Trent JC, Beach J, Burgess MA, et al. A two-arm phase II study of temozolomide in patients with advanced gastrointestinal stromal tumors and other soft tissue sarcomas. Cancer. 2003;98 (12):2693-2699. doi:10.1002/cncr.11875

15. Garcia del Muro X, Lopez-Pousa A, Martin J, et al. A phase II trial of temozolomide as a 6-week, continuous, oral schedule in patients with advanced soft tissue sarcoma: a study by the Spanish Group for Research on Sarcomas. Cancer. 2005;104(8):1706-1712. doi:10. $1002 /$ cncr.21384

16. Judson I, Radford JA, Harris M, et al. Randomised phase II trial of pegylated liposomal doxorubicin (DOXIL/CAELYX) versus doxorubicin in the treatment of advanced or metastatic soft tissue sarcoma: a study by the EORTC Soft Tissue and Bone Sarcoma Group. Eur $J$ Cancer. 2001;37(7):870-877. doi:10.1016/S0959-8049(01)00050-8
17. D’Ambrosio L, Touati N, Blay JY, et al. Doxorubicin plus dacarbazine, doxorubicin plus ifosfamide, or doxorubicin alone as a first-line treatment for advanced leiomyosarcoma: a propensity score matching analysis from the European Organization for Research and Treatment of Cancer Soft Tissue and Bone Sarcoma Group. Cancer. 2020;126(11):2637-2647. doi:10.1002/ cncr.32795

18. van der Graaf WT, Blay JY, Chawla SP, et al. Pazopanib for metastatic soft-tissue sarcoma (PALETTE): a randomised, double-blind, placebo-controlled Phase 3 trial. Lancet. 2012;379(9829):1879-1886. doi:10.1016/S0140-6736(12)60651-5

19. Chi Y, Fang Z, Hong X, et al. Safety and efficacy of anlotinib, a multikinase angiogenesis inhibitor, in patients with refractory metastatic soft-tissue sarcoma. Clin Cancer Res. 2018;24(21):5233-5238. doi:10.1158/1078-0432.CCR-17-3766

20. Chi Y, Yao Y, Wang S, et al. Anlotinib for metastasis soft tissue sarcoma: a randomized, double-blind, placebo-controlled and multi-centered clinical trial. J Clin Oncol. 2018;36(15_suppl):11503. doi:10.1200/JCO.2018.36.15_suppl.11503

21. Mir O, Brodowicz T, Italiano A, et al. Safety and efficacy of regorafenib in patients with advanced soft tissue sarcoma (REGOSARC): a randomised, double-blind, placebo-controlled, Phase 2 trial. Lancet Oncol. 2016;17(12):1732-1742. doi:10.1016/ S1470-2045(16)30507-1

22. Ferriss JS, Atkins KA, Lachance JA, Modesitt SC, Jazaeri AA. Temozolomide in advanced and recurrent uterine leiomyosarcoma and correlation with o6-methylguanine DNA methyltransferase expression: a case series. Int J Gynecol Cancer. 2010;20 (1):120-125. doi:10.1111/IGC.0b013e3181c7fe53

23. Bujko M, Kowalewska M, Danska-Bidzinska A, Bakula-Zalewska E, Siedecki JA, Bidzinski M. The promoter methylation and expression of the O6-methylguanine-DNA methyltransferase gene in uterine sarcoma and carcinosarcoma. Oncol Lett. 2012;4(3):551-555. doi:10.3892/ol.2012.771

24. Oza J, Doshi SD, Hao L, Musi E, Schwartz GK, Ingham M. Homologous recombination repair deficiency as a therapeutic target in sarcoma. Semin Oncol. 2020;47(6):380-389. doi:10.1053/j. seminoncol.2020.10.002

25. Khusnutdinov RR, Boichuk SV. Mechanisms of sensitivity of soft tissue sarcoma cells to temozolomide. Bull Exp Biol Med. 2017;163 (2):260-262. doi:10.1007/s10517-017-3779-x
Cancer Management and Research

\section{Publish your work in this journal}

Cancer Management and Research is an international, peer-reviewed open access journal focusing on cancer research and the optimal use of preventative and integrated treatment interventions to achieve improved outcomes, enhanced survival and quality of life for the cancer patient.
The manuscript management system is completely online and includes a very quick and fair peer-review system, which is all easy to use. Visit http://www.dovepress.com/testimonials.php to read real quotes from published authors. 